\title{
Pathophysiology of Diabetes Mellitus in Cushing's Syndrome
}

\author{
Rosario Pivonello Monica De Leo Pasquale Vitale Alessia Cozzolino \\ Chiara Simeoli Maria Cristina De Martino Gaetano Lombardi Annamaria Colao \\ Department of Molecular and Clinical Endocrinology and Oncology, 'Federico II' University, Naples, Italy
}

\section{Key Words}

Diabetes mellitus $\cdot$ Insulin resistance $\cdot$ Insulin sensitivity

Glucose intolerance $\cdot$ Cushing's disease $\cdot$ Hypercortisolism

\begin{abstract}
Cushing's syndrome is commonly complicated with an impairment of glucose metabolism, which is often clinically manifested as diabetes mellitus. The development of diabetes mellitus in Cushing's syndrome is both a direct and indirect consequence of glucocorticoid excess. Indeed, glucocorticoid excess induces a stimulation of gluconeogenesis in the liver as well as an inhibition of insulin sensitivity both in the liver and in the skeletal muscles, which represent the most important sites responsible for glucose metabolism. In particular, glucocorticoid excess stimulates the expression of several key enzymes involved in the process of gluconeogenesis, with a consequent increase of glucose production, and induces an impairment of insulin sensitivity either directly by interfering with the insulin receptor signaling pathway or indirectly, through the stimulation of lipolysis and proteolysis and the consequent increase of fatty acids and amino acids, which contribute to the development of insulin resistance. Moreover, the peculiar distribution of adipose tissue throughout the body, with the predominance of visceral adipose tissue, significantly contributes to the worsening of insulin resistance and the development of a metabolic syndrome, which participates in the occurrence and mainte-
\end{abstract}

nance of the impairment of glucose tolerance. Finally, glucocorticoid excess is able to impair insulin secretion as well as act at the level of the pancreatic beta cells, where it inhibits different steps of the insulin secretion process. This phenomenon is probably responsible for the passage from an impairment of glucose tolerance to an overt diabetes mellitus in susceptible patients with Cushing's syndrome.

Copyright $\odot 2010$ S. Karger AG, Basel

\section{Introduction}

Cushing's syndrome, characterized by endogenous glucocorticoid (GC) hormone excess, is associated with several systemic complications, including the impairment of glucose metabolism, which often becomes clinically manifest with the development of diabetes mellitus [1]. The prevalence of diabetes mellitus in patients with Cushing's syndrome ranges from 20 to $50 \%$, although an underestimation of this percentage is conceivable considering that the presence of diabetes mellitus is not always accurately investigated in patients with Cushing's syndrome, displaying a reduced glucose tolerance more than an increased fasting glucose level. Moreover, the prevalence of the impairment of glucose metabolism becomes close to $70 \%$ if the presence of impaired fasting glycemia or mainly impaired glucose tolerance is considered [2]. The predominant mechanisms underlying the develop-

\section{KARGER}

Fax +4161306 1234 E-Mail karger@karger.ch www.karger.com

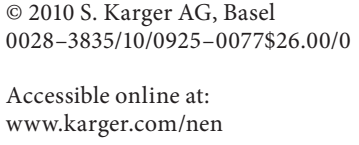

Department of Molecular and Clinical Endocrinology and Oncology

'Federico II' University of Naples, Via Sergio Pansini, 5

IT-80131 Naples (Italy)

Tel. +39 081746 4737, Fax +39 081546 5443, E-Mail rpivone@ tin.it 
ment of the impairment of glucose tolerance and diabetes mellitus in Cushing's syndrome is represented by the stimulation of gluconeogenesis and the development of insulin resistance, in association with the occurrence of an impairment of insulin secretion by the endocrine pancreas [3]. The concomitant occurrence of different metabolic complications, including dyslipidemia and impairment of protein metabolism, as well as the 'metabolic syndrome' associated with the increase and predominance of visceral adipose tissue significantly participates in the pathogenesis of insulin resistance and, consequently, the development of diabetes mellitus [3].

\section{Physiological Role of GCs on Glucose Metabolism}

GC hormones are secreted by the cortex of the adrenal gland under the control of the hypothalamus-pituitaryadrenal axis. In humans, the most important agent belonging to the GCs is represented by cortisol, whereas corticosterone is the main adrenal cortex hormone in rodents, generally used for experimental studies on the adrenal gland. GCs play an important role in glucose metabolism as well as in lipid and protein metabolism and significantly contribute to the energy homeostasis. The most important physiological role of GCs on metabolism is displayed during the postprandial period, when they behave as contra-insular hormones, providing substrates for oxidative metabolism by stimulating lipolysis and proteolysis, with consequent release of fatty acids and amino acids, and by inducing glucose production, through the stimulation of gluconeogenesis and the inhibition of glycogen synthesis. The metabolic effects of GCs, including the actions directed to the regulation of glucose metabolism, are mainly exerted not only in the liver, and in the skeletal muscle, which is responsible for the great majority of postprandial glucose uptake from the circulation and lodge the largest store of glycogen, but in the adipose tissue as well [4].

\section{Pathological Role of GC Excess on Glucose Metabolism}

GC excess induces an excessive glucose production acting mainly at the level of liver and skeletal muscle. In the liver, GCs increase glucose production both directly through the stimulation of gluconeogenesis and indirectly through the inhibition of insulin sensitivity. In the skeletal muscle, GCs increase glucose production be- cause of the development of insulin resistance, which is responsible of the decrease of glucose uptake and the inhibition of glycogen synthesis. It is noteworthy that the onset of insulin resistance is related to either a direct effect of GCs on insulin receptor signaling pathway or an indirect effect of GCs altering the insulin function through the changes in lipid and protein metabolism [5]. Figure 1 shows a schematic representation of the main effects of GCs on glucose metabolism in the liver and the skeletal muscle.

\section{Effects of GC Excess in the Liver}

In the liver, GC excess increases glucose production either directly, activating a series of genes involved in the glucose metabolism [6], or indirectly, by antagonizing the metabolic action of insulin, the most important hormone responsible for the suppression of the endogenous glucose production [7]. The direct increase of glucose production may occur in the basal state and it is probably due to different mechanisms: (1) induction of expression of essential enzymes for the gluconeogenesis [8]; (2) stimulation of lipolysis and proteolysis, with consequent increase of substrates for gluconeogenesis [9], and (3) potentiation of different hormones involved in the glucose metabolism, mainly glucagon, whose actions lead to increased glucose production [10]. The indirect increase of glucose production, obtained through the antagonism of insulin action, mainly reflects on a depression of glycogen storage in the liver [2].

\section{Effects of GC Excess in the Skeletal Muscle}

In the skeletal muscle, GC excess reduces insulin sensitivity, with a consequent increase of glucose levels in the general circulation. GC excess influences insulin sensitivity through two different mechanisms: directly, by an impairment of the insulin receptor signaling pathway, as well as indirectly, by modifying the lipid and protein metabolism [2]. This action mainly reflects on a decrease of glycogen synthesis and glucose uptake. The direct interference of GCs with the insulin receptor signaling pathway has been demonstrated mostly in animal by in vitro and in vivo studies, which confirmed that GCs do not influence insulin receptor expression but mainly induce a postreceptor defect [11]. Indeed, in rat skeletal muscle cells, dexamethasone decreased the expression and phosphorylation of crucial molecules mediating the effect of insulin receptor at intracellular levels, including the insulin receptor substrate (IRS)-1, phosphatidylinositol-3kinase (PI3K) and protein kinase B (PKB/AKT). These actions result in a decrease of the glucose transporter 


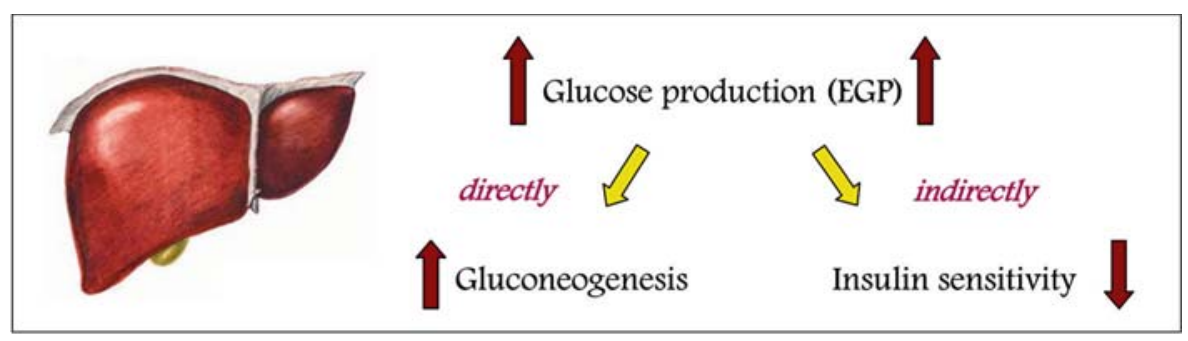

Fig. 1. Effects of GCs on glucose metabolism at the level of liver and skeletal muscle.

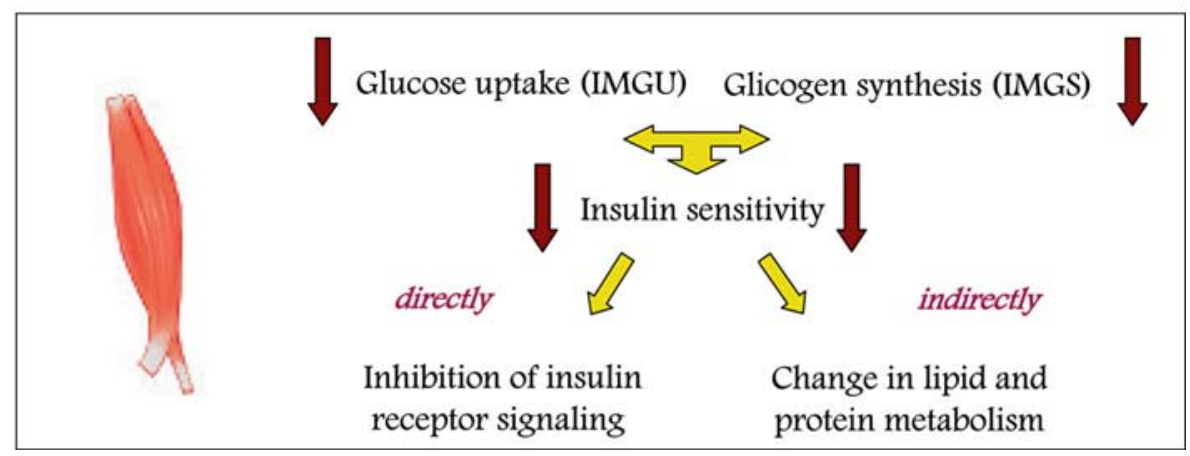

Fig. 2. Effects of GCs on the insulin receptor signaling pathways. GC indicates the processes inhibited by GC excess. IRS-1 = Insulin receptor substrate-1; $\mathrm{PI} 3 \mathrm{~K}=$ phosphatidylinositol-3-kinase; $\mathrm{PKB}=$ protein kinase B; GSK-3 = glycogen synthase kinase-3.

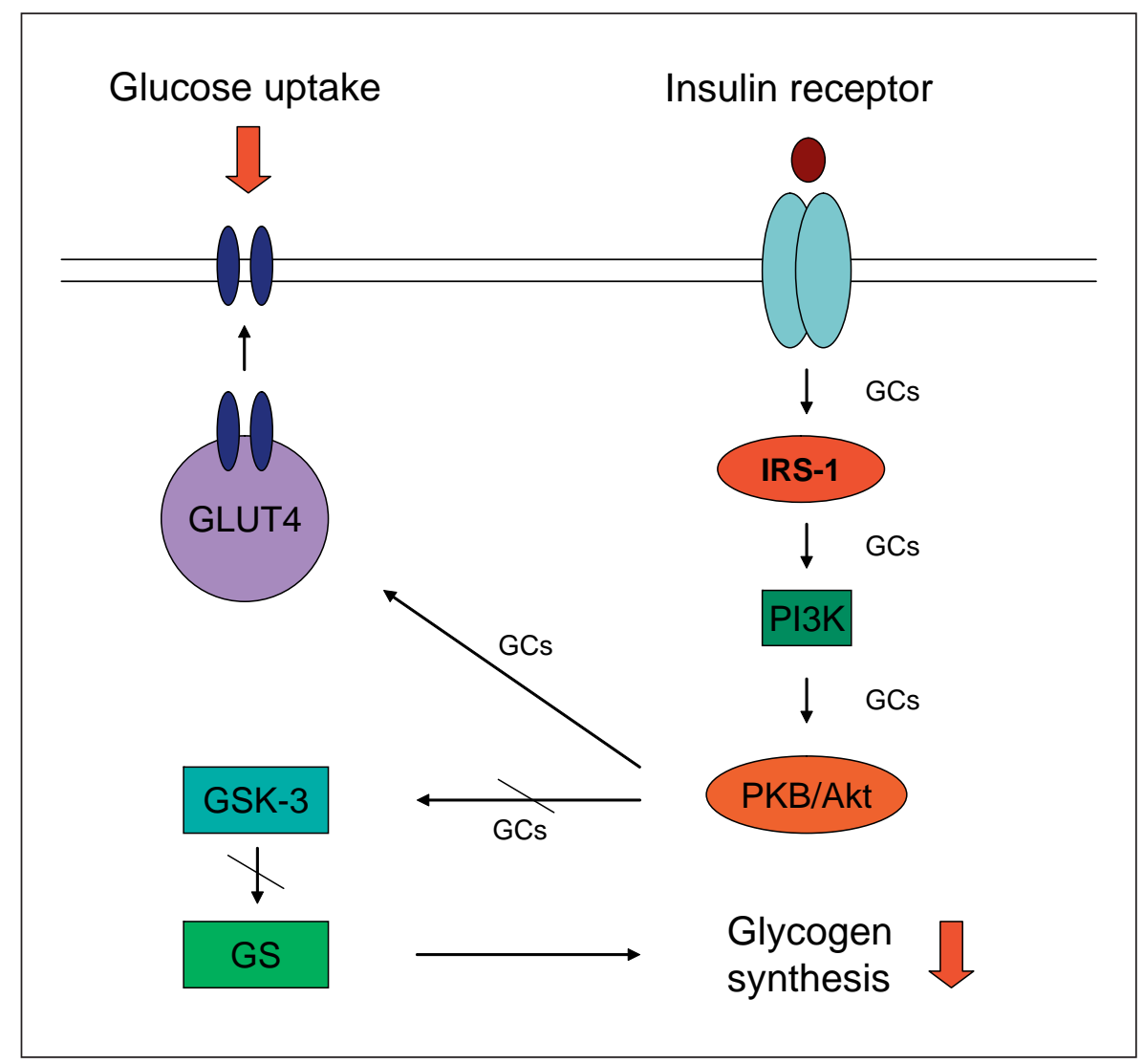


GLUT4 migration to the cell surface, with consequent reduction of glucose uptake $[12,13]$, as well as in a decrease of glycogen synthase kinase (GSK)-3 phosphorylation, with a consequent decrease of glycogen synthesis [14]. In humans, the limited number of studies on the treatment with dexamethasone in healthy subjects seem to confirm these findings. Figure 2 shows a schematic overview on the effects of GCs on the insulin receptor signaling pathway in skeletal muscle. It is noteworthy that GCs induce insulin resistance also via the changes in protein and lipid metabolism. Indeed, GC excess is associated with a decrease in protein synthesis and an increase in protein degradation, resulting in an elevation of amino acids, which impair different steps of the intracellular pathway associated with the activation of insulin receptors. Moreover, GC excess is associated with an increase of lipolysis, with a consequent elevation of fatty acids responsible for the impairment of glucose uptake and disposal [2].

\section{Effects of GC Excess in Adipose Tissue}

GCs plays a pivotal role in the regulation of adipose tissue differentiation, distribution and metabolism. It is clearly documented that GCs are able to stimulate the differentiation of pre-adipocytes into adipocytes, which are responsible for the increased body fat mass associated with GC excess. However, GCs have a remarkably differential effect in peripheral and visceral adipose tissue since they enhance the visceral fat specifically, whereas they display a relatively shrinking effect on peripheral fat, determining the typical central obesity associated with Cushing's syndrome [15]. In addition, GCs significantly affect adipose tissue metabolism, influencing the synthesis and release of different hormones, mainly adipokines, which contribute to the development of insulin resistance [16]. The occurrence of insulin resistance in adipose tissue, together with the predominance of visceral or peripheral adipose tissue, participate in the pathogenesis of the 'metabolic syndrome', which is associated with the development of the impairment of glucose metabolism.

\section{Effects of GC Excess on Insulin Secretion}

GC excess has been found to influence insulin secretion by the pancreatic beta cells, which physiologically play a pivotal role in glucose metabolism and are responsible for the adaptation of insulin secretion to the peripheral insulin sensitivity in order to maintain normal circulating glucose levels [17]. The role of GC excess on insulin secretion has been discussed for a long time. The data collected in the literature on this issue suggest that the effect of GCs on pancreatic beta cell function are highly dependent on the type and dose of the GCs, the duration of exposure as well as the susceptibility of the population exposed to GCs. The majority of the studies, however, have been performed in animals. The in vitro administration of GCs in pancreatic beta cells derived from rodents has been demonstrated to decrease insulin release, probably acting at the level of different steps of the insulin secretion process, starting from glucose uptake and utilization and ending with calcium fluxes and exocytosis of granules containing insulin [2]. Indeed, GCs have been documented to reduce the expression of the glucose transporter GLUT2 [18] and glucokinase [19], the most important enzymes responsible for the start of glucose oxidation and the activation of the energy metabolism essential for the secretion process. Moreover, dexamethasone has been found to increase the inward potassium current through the upregulation of potassium channels, induced by the activation of serum- and glucocorticoid-inducible kinase (SGK)-1 [20]. In addition, GC excess seems to influence the signaling pathway and the effect of different insulin secretagogues. The in vivo administration of GCs in rodents confirms the inhibiting effects of GCs on insulin secretion, although this effect seems to be induced more after acute than after chronic administration [2]. In humans, the acute administration of GCs impairs insulin secretion, with a consequent increase in glucose levels [21]. However, an increase in insulin secretion has been demonstrated with the clamp technique in normal subjects after short-term treatment with GCs, suggesting a compensation mechanism by the pancreatic beta cells for the peripheral insulin resistance induced by GCs [22], although contradictory data have been produced using more physiological procedures [20]. The long-term treatment with GCs seems to induce a pancreatic beta cell dysfunction, probably as a consequence of both the inhibition of insulin synthesis and secretion, as well as apoptosis of the pancreatic beta cells induced directly and indirectly by GCs with a consequent loss of beta cell function and the development of diabetes mellitus, especially in susceptible individuals [2].

\section{Conclusions}

GC excess is associated with an increased glucose production, due to the stimulation of gluconeogenesis and development of insulin resistance mainly in the liver and in the skeletal muscle, which reduces glycogen synthesis and glucose uptake. The mechanisms underlying this 
phenomenon are represented by the impairment of the insulin receptor signaling cascade, operated by GCs directly or indirectly via the stimulation of lipolysis and proteolysis. The effects of GCs on adipose tissue, represented by the increase of visceral obesity, contribute to the development of the metabolic syndrome, worsening the insulin resistance. The totality of these effects induce an impairment of glucose tolerance. The concomitant inhibitory effect of GCs on insulin secretion at the pancre- atic beta cell level is probably responsible, especially in susceptible subjects, for the passage from the impairment of glucose tolerance to an overt diabetes mellitus in patients with Cushing's syndrome.

\section{Disclosure Statement}

The authors have nothing to disclose.

\section{References}

$\checkmark 1$ Pivonello R, De Martino MC, De Leo M, Lombardi G, Colao A: Cushing's syndrome. Endocrinol Metab Clin North Am 2008;37: 135-149.

$\checkmark 2$ Resmini E, Minuto F, Colao A, Ferone D Secondary diabetes associated with principal endocrinopathies: the impact of new treatment modalities. Acta Diabetol 2009; 46:85-95.

3 van Raalte DH, Ouwens DM, Diamant M: Novel insights into glucocorticoid-mediated diabetogenic effects: towards expansion of therapeutic options? Eur J Clin Invest 2009; 39:81-93.

4 McMahon M, Gerich J, Rizza R: Effects of glucocorticoids on carbohydrate metabolism. Diabetes Metab Rev 1988;4:17-30.

5 Andrews RC, Walker BR: Glucocorticoids and insulin resistance: old hormones, new targets. Clin Sci 1999;96:513-523.

-6 Vegiopoulos A, Herzig S: Glucocorticoids, metabolism and metabolic diseases. Mol Cell Endocrinol 2007;275:43-61.

7 Rooney DP, Neely RD, Cullen C, et al: The effect of cortisol on glucose/glucose-6-phosphate cycle activity in insulin action. J Clin Endocrinol Metab 1993;77:1180-1183.

$\checkmark 8$ Vander Kooi BT, Onuma H, Oeser JK, et al: The glucose-6-phosphatase catalytic subunit gene promoter contains both positive and negative glucocorticoid response elements. Mol Endocrinol 2005;19:3001-3022.

$>$ Kraus-Friedman N: Hormonal regulation of hepatic gluconeogenesis. Physiol Rev 1984; 64:170-259.
10 Dirlewanger M, Schneiter PH, Paquot N, Jequier E, Rey V, Tappy L: Effects of glucocorticoids on hepatic sensitivity to insulin and glucagon in man. Clin Nutr 2000;19:29-34.

11 Rizza RA, Mandarino LJ, Gerich JE: Cortisol-induced insulin resistance in man: impaired suppression of glucose production and stimulation of glucose utilization due to a postreceptor defect of insulin action. J Clin Endocrinol Metab 1982;54:131-138.

12 Saad MJ, Folli F, Kahn JA, Kahn CR: Modulation of insulin receptor, insulin receptor substrate-1, and phosphatidylinositol 3-kinase in liver and muscle of dexamethasonetreated rats. J Clin Invest 1993;92:20652072

13 Weinstein SP, Wilson CM, Pritsker A, Cushman SW: Dexamethasone inhibits insulinstimulated recruitment of GLUT4 to the cell surface in rat skeletal muscle. Metabolism 1998;47:3-6.

14 Ruzzin J, Wagman AS, Jensen J: Glucocorticoid-induced insulin resistance in skeletal muscles: defects in insulin signalling and the effects of a selective glycogen synthase kinase-3 inhibitor. Diabetologia 2005;48: 2119-2130.

15 Seckl JR, Morton NM, Chapman KE, Walker BR: Glucocorticoids and 11beta-hydroxysteroid dehydrogenase in adipose tissue. Recent Prog Horm Res 2004;59:359-393.
16 Kershaw EE, Flier IS: Adipose tissue as an endocrine organ. J Clin Endocrinol Metab 2004;89:2548-2556.

17 Kahn SE: The relative contributions of insulin resistance and beta cell dysfunction to the pathophysiology of type 2 diabetes. Diabetologia 2003;46:3-19.

18 Gremlich S, Roduit R, Thorens B: Dexamethasone induces posttranslational degradation of GLUT2 and inhibition of insulin secretion in isolated pancreatic beta cells. Comparison with the effects of fatty acids. J Biol Chem 1997;272:3216-3222.

19 Borboni P, Porzio O, Magnaterra R, et al: Quantitative analysis of pancreatic glucokinase gene expression in cultured beta cells by competitive polymerase chain reaction. Mol Clin Endocrinol 1996;117:175-181.

20 Ullrich S, Berchtold S, Ranta F, et al: Serum- and glucocorticoid-inducible kinase 1 (SGK1) mediates glucocorticoid-induced inhibition of insulin secretion. Diabetes 2005; 54:1090-1099.

21 van Raalte D, Nofrate V, Bunck M, Van Iersel T, Elassaiss Schaap J, Nãssander U, Heine R, Mari A, Dokter W, Diamant M: Acute and two-week exposure to prednisolone impair different aspects of beta-cell function in healthy men. Eur J Endocrinol 2010;162: 729-735.

-22 Matsumoto K, Yamasaki H, Akazawa S, et al: High dose but not low dose dexamethasone impairs glucose tolerance by inducing compensatory failure of pancreatic beta cells in normal men. J Clin Endocrinol Metab 1996; 81:2621-2626. 